\title{
EFEITO DO PLANO DE NUTRIČ̃̃O SOBRE O RENDIMENTO DE CARCAÇA DE CODORNAS TIPO CARNE
}

\author{
Effect of nutrition plane on the carcass yield of quails meat type
}

\author{
Edson Lindolfo da Silva ${ }^{1}$, José Humberto Vilar da Silva², José Jordão Filho ${ }^{3}$, \\ Marcelo Luís Gomes Ribeiro ${ }^{3}$
}

\begin{abstract}
RESUMO
Com o objetivo de avaliar o efeito do plano de nutrição (PN) e do sexo (S) sobre rendimento de carcaça de codornas utilizouse 576 codornas, distribuídas num delineamento inteiramente ao acaso em arranjo fatorial 9 X 2 (PN X S), que resultaram em 18 tratamentos com quatro repetições de oito aves. Os PN foram avaliados de 1 a 21 e de 22 a 42 dias de idade: $\mathrm{PN}_{1}=28 \%$ PB, 1,5\% de lisina (L) e $1,1 \%$ de metionina+cistina (MC), e $24 \%$ de PB, $1,3 \%$ L e $0,9 \% \mathrm{MC} ; \mathrm{PN}_{2}=25,2 \% \mathrm{~PB}, 1,23 \%$ L e $0,83 \% \mathrm{MC}$, e $21,6 \%$ $\mathrm{PB}, 1,0 \% \mathrm{~L}$ e $0,74 \% \mathrm{MC} ; \mathrm{PN}_{3}=22,4 \% \mathrm{~PB}, 1,2 \% \mathrm{~L}$ e $0,71 \% \mathrm{MC}$, e $19,2 \% \mathrm{~PB}, 0,95 \% \mathrm{~L}, 0,64 \% \mathrm{MC} ; \mathrm{PN}_{4}=\mathrm{PN}_{2}+\mathrm{L} ; \mathrm{PN}_{5}=\mathrm{PN}_{3}+\mathrm{L}$; $\mathrm{PN}_{6}=\mathrm{PN}+\mathrm{MC} ; \mathrm{PN}_{7}=\mathrm{PN}_{3}+\mathrm{MC} ; \mathrm{PN}_{8}=\mathrm{PN}_{2}+\mathrm{L}+\mathrm{MC}$ e $\mathrm{PN}_{9}=\mathrm{PN}_{3}+\mathrm{L}+\mathrm{MC}$. As suplementações com fontes industriais de Lys $\mathrm{e}$ Met+Cys foram realizadas para garantir níveis semelhantes destes aminoácidos nas dietas em que a proteína foi reduzida (do $\mathrm{PN}_{4}$ até o $\mathrm{PN}_{9}$ ). Houve interações do PN com o sexo para o peso vivo, peso de carcaça, peito e pernas. Para a fase de 1 a 21 recomendam-se $22,4 \%$ de $\mathrm{PB}, 1,2 \%$ de lisina e 1,1\% de aminoácidos sulfurosos (AAS), e de 22 a 42 sugerem-se 19,2\% de PB, 0,95\% de lisina e $0,9 \%$ de AAS.
\end{abstract}

Termos para indexação: Avaliação de carcaça, codornas européias, exigência nutricional.

\section{ABSTRACT}

This work was carried out with the objective to evaluate the effect of the nutrition plane (NP) and sex (S) on carcass yield of 576 meat type quails, distributed in the completely randomized design in factorial arrangement 9 X 2 (NP X S), that resulted in 18 treatments with four replicates of eight quails. The NP evaluating from 1 to 21 and from 22 to 42 days of age were: $\mathrm{NP} \mathrm{P}_{1}=28 \% \mathrm{CP}$, $1.5 \%$ of lysine (L) and $1.1 \%$ of metionine+cystine $(\mathrm{MC})$, and $24 \%$ of $\mathrm{CP}, 1.3 \% \mathrm{~L}$ and $0.9 \% \mathrm{MC} ; \mathrm{NP}_{2}=25.2 \% \mathrm{CP}, 1.23 \% \mathrm{~L}$ and $0.83 \% \mathrm{MC}$, and $21.6 \% \mathrm{CP}, 1.0 \% \mathrm{~L}$ and $0.74 \% \mathrm{MC} ; \mathrm{NP}=22,4 \% \mathrm{CP}, 1.2 \% \mathrm{~L}$ and $0.71 \% \mathrm{MC}$, and $19.2 \% \mathrm{~PB}, 0.95 \% \mathrm{~L}, 0.64 \% \mathrm{MC}$; $\mathrm{NP}_{4}=\mathrm{NP}_{2}+\mathrm{L} ; \mathrm{NP}_{5}=\mathrm{NP}_{3}+\mathrm{L} ; \mathrm{NP}_{6}=\mathrm{NP}_{2}+\mathrm{MC} ; \mathrm{NP}_{7}=\mathrm{NP}_{3}+\mathrm{MC} ; \mathrm{NP}_{8}=\mathrm{NP}_{2}+\mathrm{L}+\mathrm{MC}$ and $\mathrm{NP}_{9}=\mathrm{NP}_{3}+\mathrm{L}+\mathrm{MC}$. The supplementations with industrial sources of Lys and Met+Cys were donne to guarantee levels similar of these amino acids in the diets when the protein was reduced (from $\mathrm{NP}_{4}$ to $\mathrm{NP}_{9}$ ). There were interactions of $\mathrm{NP}$ with the sex for the live weight, carcass weight, breast and drumstick. From 1 to 21 days of age recommended $22.4 \%$ of $\mathrm{CP}, 1.2 \%$ of lysine and $1.1 \%$ of sulfurous amino acids (SAA), and from 22 to 42 days old should be $19.2 \%$ of PB, $0.95 \%$ of lysine and $0.9 \%$ of SAA.

Index terms: Carcass evaluation, european quails, nutritional requirements.

\section{(Recebido em 9 de agosto de 2005 e aprovado em 27 de março de 2006)}

\section{INTRODUÇÃO}

A oferta de carne de codornas pode, em curto prazo de tempo, se converter em importante fonte alternativa de proteína para o consumo humano. Fatores como o pequeno investimento inicial, a alta resistência das aves às enfermidades e o baixo consumo absoluto de ração, contribuem para estimular a criação dessas aves. Entretanto, as informações disponíveis na literatura sobre a nutrição de codornas ainda são escassas.

Grande parte da população mundial e, especialmente a brasileira, está caminhando para se tornar consumidor de perfil mais exigente, não só em termos de sabor, mas também no que se refere a qualidade nutritiva e sanitária dos alimentos que consome, dispondo-se a pagar preços mais elevados por produtos comprovadamente superiores (OLIVEIRA et al., 2000). Sob este aspecto, a carne de codornas pode ser apresentada como um produto diferenciado, altamente palatável que permite vários tipos de processamentos, como a elaboração de conservas, defumados e assados (PANDA \& SINGH, 1990).

A produção de carne de codornas foi por longo período de tempo, caracterizada pelo abate de aves provenientes das criações destinadas à produção de ovos, onde os machos eram criados até os 42 ou 49 dias de idade e depois eram abatidos. Por sua vez, as fêmeas permaneciam no plantel até um ano de idade, quando encerravam o

\footnotetext{
${ }^{1}$ Licenciatura em Ciências Agrárias, Doutorando em Zootecnia da Universidade Federal de Lavras/UFLA - Cx. P. 3037 - $37.200-000$ - Lavras, MG Bolsista CNPq -edsonlindolfo@yahoo.com.br

${ }^{2}$ Zootecnista, Professor do Departamento de Agropecuária da Universidade Federal da Paraíba/UFPB - Cx. P. 07 - 58.220-000 - Bananeiras, PB Bolsista CNPq - jvilar@cft.ufpb.br

${ }^{3}$ Licenciatura em Ciências Agrárias - Programa de Doutorando Integrado em Zootecnia - Professor do Departamento de Agropecuária da Universidade Federal da Paraíba/UFPB - Cx. P. 07 - 58.220-000 - Bananeiras, PB - Bolsista Capes - jjordaofilho@yahoo.com.br; marcelo@cft.ufpb.br
} 
primeiro ciclo de produção de ovos (GARCIA, 2002). Em conseqüência, as carcaças obtidas com o abate dos machos eram muito pequenas, pesando entre 70 e 110 gramas, e a carne das fêmeas era relativamente dura por se tratar de aves de aproximadamente um ano de idade.

No mercado brasileiro, surgiram há algum tempo alguns tipos de codornas mais pesadas que podem atender melhor aos requisitos necessários à produção de carnes. Não se sabe ao certo sua procedência, contudo, fenotipicamente, essas aves são muito semelhantes às codornas japonesas (Coturnix coturnix japonica), entretanto apresentam peso vivo compreendido entre 200 e 300 gramas, temperamento mais calmo, maior peso de ovos e maturidade sexual semelhante ao das codornas destinadas à produção de ovos. No Brasil, a única empresa que produz e comercializa codornas para corte apresentou um crescimento de $35 \%$ nas vendas do segmento de aves exóticas no período 1991 a 1995 , sendo que no período de 1996 e 1997 houve estabilização das vendas devido à falta do produto para atender a demanda dos mercados interno e externo e, em 1998 houve um aumento de 60\% na produção de codornas. Essas aves são comercializadas em embalagens contendo aproximadamente cinco carcaças congeladas de peso médio aproximado de $200 \mathrm{~g}$, ao passo que nos Estados Unidos, além desse tipo de carcaça, são comercializadas codornas desossadas da espécie americana ou Bobwhite Quail (Colinus virginianus), com pesos de até 300 gramas pósprocessamento (MURAKAMI et al., 1998).

A qualidade da carne de codornas é reconhecida desde os povos mais antigos, por seu alto conteúdo em proteínas e por sua escassa infiltração de gordura, aliada a rapidez do ciclo de crescimento, considerado em média de 35 dias para atingir a fase adulta, proporcionando uma carne muito tenra, com preparação gastronômica fácil e rápida, constituindo-se numa carne superior as outras (DALMAU, 2002). Esta qualidade é fortemente influenciada pelo peso e proporção de peito, o principal e mais valorizado componente da carcaça de codornas. Escassos estudos em condições brasileiras têm sido realizados para desenvolver planos de nutrição para codornas do tipo carne, baseados na redução de proteína bruta da ração. Portanto, objetivou-se neste trabalho avaliar o efeito do plano de nutrição e do sexo sobre os rendimentos de carcaça e de cortes nobres em codornas de 1 a 42 dias de idade, criadas para a produção de carne.

\section{MATERIAL E MÉTODOS}

O experimento foi realizado no aviário experimental do Setor de Pesquisa em Nutrição de Aves - SPNA do Departamento de Agropecuária do Centro de Formação de Tecnólogos (CFT), Campus III da UFPB, na cidade de Bananeiras, microrregião do brejo paraibano.

Foram alojadas 576 codornas européias (Coturnix coturnix coturnix L), de um dia de idade, sendo 288 de cada sexo, em boxe com piso coberto com cama de maravalha. As aves foram distribuídas num delineamento inteiramente ao acaso em esquema fatorial 9 X 2 (nove planos de nutrição X sexo), resultando em 18 tratamentos com quatro repetições de 8 codornas cada um. Os planos de nutrição (PN) foram definidos com base na redução da proteína da ração em 10 e $20 \%$ dos níveis recomendados por (LEESON et al., 1997) de 1 a 21 dias de idade e, os níveis preconizados por (SHRIVASTAV \& PANDA, 1999) de 22 a 42 dias de idade (Tabelas 1 e 2) e suplementação com L-Lisina $\cdot \mathrm{HCl}(78,4 \%)$ e DL-Metionina (99\%) para atender níveis de lisina e/ou metionina+cistina semelhantes aos destes aminoácidos presentes nas dietas do $\mathrm{PN}_{1}$.

As aves foram alimentadas com níveis nutricionais definidos da seguinte forma:

Planos de nutrição de 1 a 21 dias

(Nutrition plan of 1 at 21 days)

$\begin{array}{ll}\mathrm{PN}_{1} \text { (controle) }= & 28 \% \mathrm{~PB} ; 1,5 \% \mathrm{~L} \text { e } 1,1 \% \mathrm{MC} \\ \mathrm{PN}_{2}= & 25,2 \% \mathrm{~PB} ; 1,23 \% \mathrm{~L} \text { e } 0,83 \% \mathrm{MC} \\ \mathrm{PN}_{3}= & 22,4 \% \mathrm{~PB}, 1,2 \% \mathrm{~L} \text { e } 0,71 \% \mathrm{MC} \\ \mathrm{PN}_{4}= & \mathrm{PN}_{2}+0,22 \% \mathrm{~L} \\ \mathrm{PN}_{5}= & \mathrm{PN}_{3}+0,30 \% \mathrm{~L} \\ \mathrm{PN}_{6}= & \mathrm{PN}_{2}+0,23 \% \mathrm{MC} \\ \mathrm{PN}_{7}= & \mathrm{PN}_{3}+0,39 \% \mathrm{MC} \\ \mathrm{PN}_{8}= & \mathrm{PN}_{2}+0,22 \% \mathrm{~L}+0,23 \% \mathrm{MC} \\ \mathrm{PN}_{9}= & \mathrm{PN}_{3}+0,30 \% \mathrm{~L}+0,39 \% \mathrm{MC}\end{array}$

Planos de nutrição de 22 a 42 dias (Nutrition plan of 22 at 42 days)

$\begin{array}{ll}\mathrm{PN}_{1}= & 24 \% \mathrm{~PB} ; 1,3 \% \mathrm{~L} \text { e } 0,9 \% \mathrm{MC} \\ \mathrm{PN}_{2}= & 21,6 \% \mathrm{~PB} ; 1,0 \% \mathrm{~L} \text { e } 0,74 \% \mathrm{MC} \\ \mathrm{PN}_{3}= & 19,2 \% \mathrm{~PB} ; 0,95 \% \mathrm{~L} \text { e } 0,64 \% \mathrm{MC} \\ \mathrm{PN}_{4}= & \mathrm{PN}_{2}+0,30 \% \mathrm{~L} \\ \mathrm{PN}_{5}= & \mathrm{PN}_{3}+0,35 \% \mathrm{~L} \\ \mathrm{PN}_{6}= & \mathrm{PN}_{2}+0,16 \% \mathrm{MC} \\ \mathrm{PN}_{7}= & \mathrm{PN}_{3}+0,26 \% \mathrm{MC} \\ \mathrm{PN}_{8}= & \mathrm{PN}_{2}+0,30 \% \mathrm{~L}+0,16 \% \mathrm{MC} \\ \mathrm{PN}_{9}= & \mathrm{PN}_{3}+0,35 \% \mathrm{~L}+0,26 \% \mathrm{MC}\end{array}$




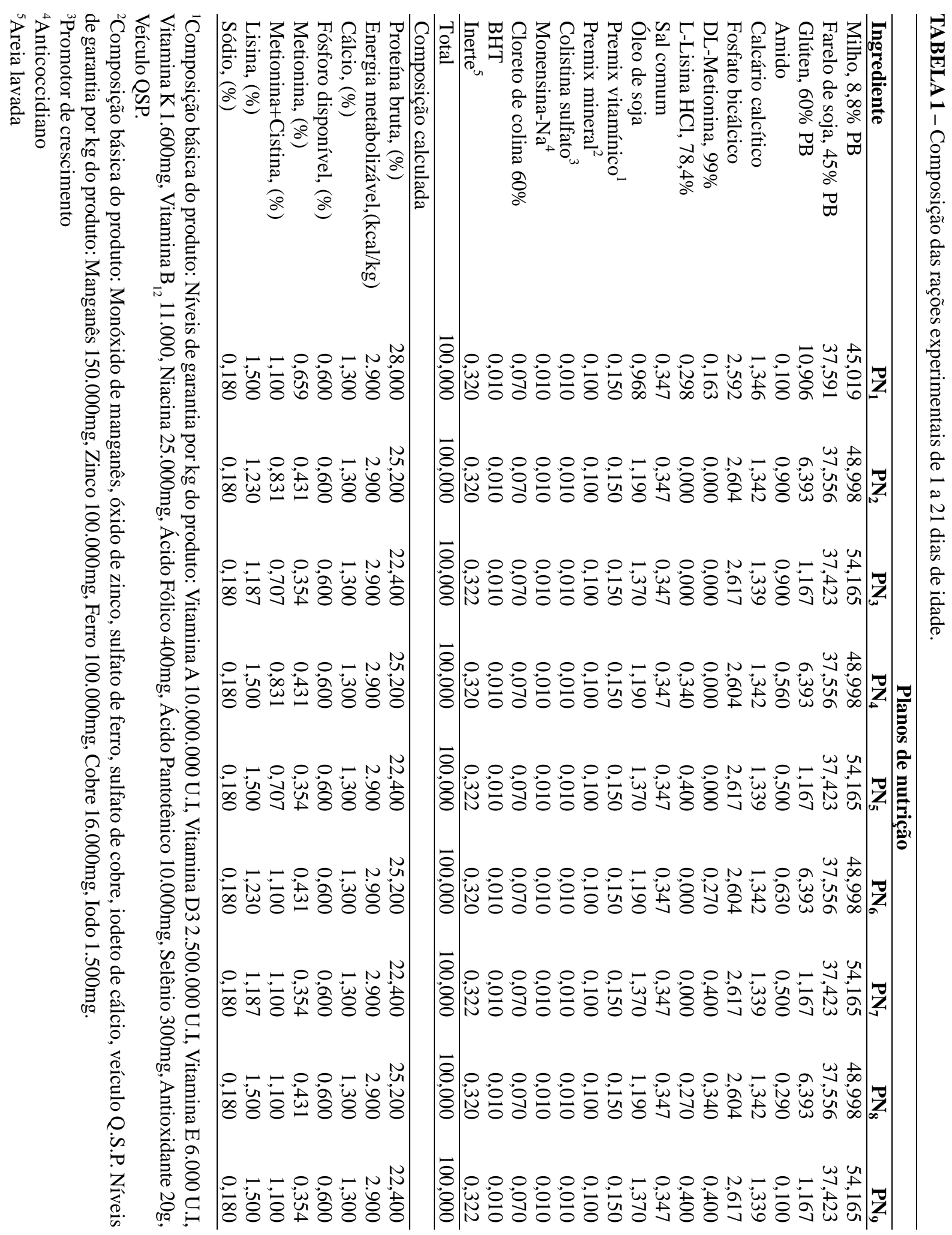

Ciênc. agrotec., Lavras, v. 31, n. 2, p. 514-522, mar./abr., 2007 


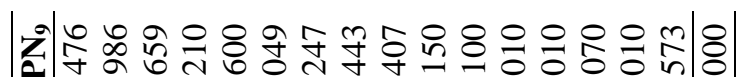

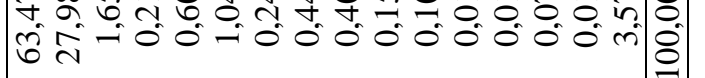

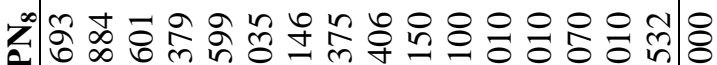

\& \& ब

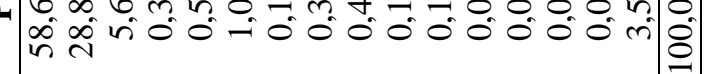

\& \& ৪

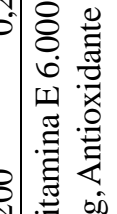

8
0
0
0
0
0
0
0
0
0
0
0

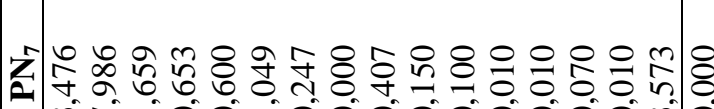

ชิ

年

(1)

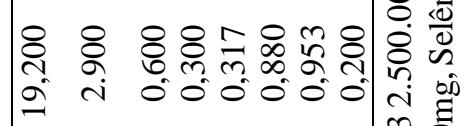
ข

:

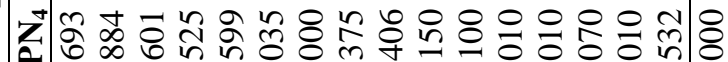

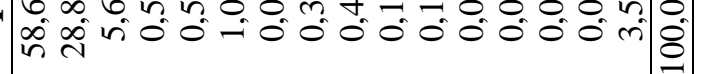

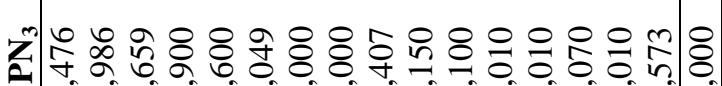
ชิ

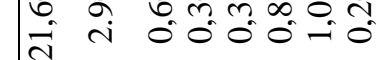

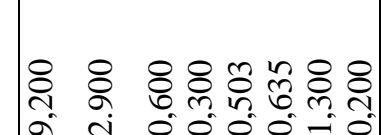

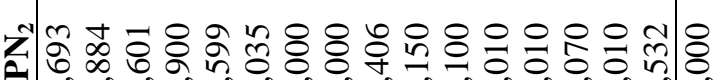

\& \& \& \&

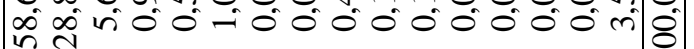

ते

\section{خ棬}

\& \& \& \&

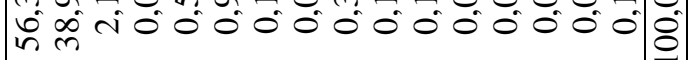

ते 
Aos 42 dias, quatro aves foram selecionadas, com \pm $5 \%$ do peso vivo médio mensurado na parcela experimental, pesadas e submetidas a um jejum alimentar de 8 horas e, em seguida, foram novamente pesadas e abatidas, depenadas, evisceradas e cortadas para as avaliações do rendimento de carcaça em relação ao peso do animal vivo, e dos rendimentos dos cortes em relação ao peso da carcaça. Foram estudados os pesos vivo $(\mathrm{g})$, peso $(\mathrm{g})$ e rendimento de carcaça $(\%)$, peso $(\mathrm{g})$ e rendimento de peito e de pernas $(\%)$.

Os dados foram analisados utilizando-se o Sistema para Análises Estatísticas e Genéticas - SAEG, desenvolvido pela Universidade Federal de Viçosa - MG (UFV, 1983). Quando o teste F apresentou interação significativa $\left(\mathrm{P}_{\leq} 0,05\right)$, os resultados das variáveis foram desdobrados nos efeitos de tratamento $\mathrm{X}$ sexo. Em caso de interação não-significativa, as médias de tratamento e sexo foram comparados, respectivamente pelo teste SNK $(\mathrm{P} \leq 0,05)$ e $\mathrm{F}(\mathrm{P} \leq 0,05)$.

\section{RESULTADOS E DISCUSSÃO}

As médias de temperatura e umidade relativa do ar mínimas e máximas obtidas foram, respectivamente, de 22 e $25{ }^{\circ} \mathrm{C}$ e 82 e $87 \%$, registradas no interior do galpão em aparelho termo higrômetro em dois períodos diários, as oito e às dezesseis horas.

Houve interações entre o plano de nutrição e o sexo das codornas para o peso vivo e os pesos de carcaça $(\mathrm{P} \leq 0,05)$, conforme a Tabela 3 .

TABELA 3 - Peso vivo, peso e porcentagem de carcaça em função do plano de nutrição (PN) e sexo de codornas criadas para a produção de carne.

\begin{tabular}{|c|c|c|c|c|c|}
\hline \multirow[b]{2}{*}{ PN } & \multicolumn{2}{|c|}{ Peso vivo (g) } & \multicolumn{2}{|c|}{ Carcaça (g) } & \multirow{2}{*}{$\begin{array}{c}\text { Carcaça }(\%) \\
\text { Média }\end{array}$} \\
\hline & Machos & Fêmeas & Machos & Fêmeas & \\
\hline $\mathrm{PN}_{1}$ & $237,25^{\mathrm{abB}}$ & $269,78^{\mathrm{aA}}$ & $170,66^{\mathrm{B}}$ & $184,81^{\mathrm{aA}}$ & 69,65 \\
\hline $\mathrm{PN}_{2}$ & $222,66^{\mathrm{abB}}$ & $252,46^{\mathrm{aA}}$ & 169,30 & $166,58^{\mathrm{ab}}$ & 69,71 \\
\hline $\mathrm{PN}_{3}$ & $226,95^{\mathrm{ab}}$ & $217,61^{b}$ & 160,79 & $157,67^{\mathrm{b}}$ & 71,56 \\
\hline $\mathrm{PN}_{4}$ & $228,05^{\mathrm{abB}}$ & $250,96^{\mathrm{aA}}$ & $161,27^{\mathrm{B}}$ & $176,24^{\mathrm{abA}}$ & 71,35 \\
\hline $\mathrm{PN}_{5}$ & $212,66^{\mathrm{bA}}$ & $189,14^{\mathrm{cB}}$ & $156,85^{\mathrm{A}}$ & $139,09^{\mathrm{cB}}$ & 71,93 \\
\hline $\mathrm{PN}_{6}$ & $241,07^{\mathrm{a}}$ & $255,30^{\mathrm{a}}$ & 173,52 & $174,37^{\mathrm{ab}}$ & 69,51 \\
\hline $\mathrm{PN}_{7}$ & $230,35^{\mathrm{abB}}$ & $264,88^{\mathrm{aA}}$ & 162,51 & $172,19^{\mathrm{ab}}$ & 69,12 \\
\hline $\mathrm{PN}_{8}$ & $230,56^{\mathrm{abB}}$ & $267,93^{\mathrm{aA}}$ & 161,45 & $171,20^{\mathrm{ab}}$ & 68,85 \\
\hline $\mathrm{PN}_{9}$ & $236,08^{\mathrm{abB}}$ & $263,27^{\mathrm{aA}}$ & 167,07 & $178,32^{\mathrm{ab}}$ & 71,11 \\
\hline Machos & \multicolumn{2}{|c|}{229,51} & \multicolumn{2}{|c|}{164,82} & $72,24^{\mathrm{a}}$ \\
\hline Fêmeas & \multicolumn{2}{|c|}{247,92} & \multicolumn{2}{|c|}{168,94} & $68,38^{b}$ \\
\hline \multicolumn{6}{|l|}{ Anova } \\
\hline $\mathrm{PN}$ & \multicolumn{2}{|c|}{$* *$} & \multicolumn{2}{|c|}{$* *$} & ns \\
\hline Sexo $(S)$ & \multicolumn{2}{|c|}{$* *$} & \multicolumn{2}{|c|}{$\mathrm{ns}$} & $* *$ \\
\hline $\mathrm{PN} * \mathrm{~S}$ & \multicolumn{2}{|c|}{$* *$} & \multicolumn{2}{|c|}{$* *$} & $\mathrm{~ns}$ \\
\hline $\mathrm{CV}(\%)$ & \multicolumn{2}{|c|}{4,742} & \multicolumn{2}{|c|}{4,079} & 3,240 \\
\hline
\end{tabular}

${ }^{1} \mathrm{PN}=$ Plano de nutrição $1=28 \% \mathrm{~PB}, 1,5 \%$ lisina (L) e 1,1\% metionina+cistina (MC) de 1 a 21 dias e $24 \% \mathrm{~PB}, 1,3 \% \mathrm{~L}$ e $0,9 \% \mathrm{MC}$ de 22 a 42 dias; $\mathrm{PN}_{2}=25,2 \% \mathrm{~PB}, 1,23 \% \mathrm{~L}$ e $0,83 \% \mathrm{MC}$ de 1 a 21 dias e de $21,6 \% \mathrm{~PB}, 1,0 \% \mathrm{~L}$ e $0,74 \%$ MC de 22 a 42 dias; $\mathrm{PN}_{3}=22,4 \% \mathrm{~PB}, 0,71 \% \mathrm{MC}$ de 1 a 21 dias e $1,2 \% \mathrm{~L}$ e $19,2 \% \mathrm{~PB}, 0,64 \% \mathrm{MC}$ e $0,95 \% \mathrm{~L}$ de 22 a 42 dias; $\mathrm{PN}_{4}=$ $\mathrm{PN}_{2}+\mathrm{L} ; \mathrm{PN}_{5}=\mathrm{PN}_{3}+\mathrm{L} ; \mathrm{PN}_{6}=\mathrm{PN}_{2}+\mathrm{MC} ; \mathrm{PN}_{7}=\mathrm{PN}_{3}+\mathrm{MC} ; \mathrm{PN}_{8}=\mathrm{PN}_{2}+\mathrm{L}+\mathrm{MC} ;$ e $\mathrm{PN}_{9}=\mathrm{PN}_{3}+\mathrm{L}+\mathrm{MC}$

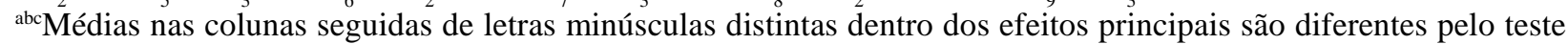
SNK $(\mathrm{P} \leq 0,05)$.

${ }^{A B}$ Médias nas linhas seguidas de letras maiúsculas distintas são diferentes pelo teste $\mathrm{F}\left(\mathrm{P}_{\leq} 0,05\right)$. 
Analisando o efeito dos planos de nutrição dentro de cada sexo, maior peso vivo para os machos foi constatado no $\mathrm{PN}_{6}$, em que as aves receberam rações com níveis intermediários de $\mathrm{PB}+\mathrm{MC}$ (25,2 e 21,6\%), nas fases de 1 a 21 e de 22 a 42 dias de idade, respectivamente, em relação ao $\mathrm{PN}_{5}$, que foram usadas dietas de baixa proteína nas fases inicial e crescimento (22,4 e 19,2\% PB) suplementadas apenas com lisina (Tabela 3). Este resultado discorda daquele observado por Oliveira (2001) que não observou efeito da redução da PB de $26 \%$ para 24, 22 e $20 \%$ sobre o peso vivo de codornas machos de 1 a 49 dias de idade.

Os maiores pesos para as fêmeas foram observados nos $\mathrm{PN}_{1}$ (dieta controle), $\mathrm{PN}_{2}, \mathrm{PN}_{4}(22,4$ e 19,2\% $\mathrm{PB}+\mathrm{L})$, $\mathrm{PN}_{6}, \mathrm{PN}_{7}, \mathrm{PN}_{8}$ e $\mathrm{PN}_{9}$ em comparação com os planos de nutrição que utilizaram as rações de baixa proteína não suplementada $\left(\mathrm{PN}_{3}\right)(22,4$ e $19,2 \% \mathrm{~PB})$ ou suplementada com lisina $\left(\mathrm{PN}_{5}\right)$, sendo que este último influenciou mais negativamente o peso das aves (P d" 0,05$)$. Os resultados do presente trabalho não concordam com os verificados por Oliveira (2001), que não constatou efeito da redução do nível de proteína da ração de $26 \%$ para 24,22 e $20 \%$ sobre o peso vivo de codornas fêmeas até 49 dias de idade. Comparações entre os dois sexos mostraram que as fêmeas apresentaram maior peso vivo nos PN 1; 2; 4; 7; 8; 9 e menor no $\mathrm{PN}_{5}$ em comparação com os machos. Variando a proteína da dieta de 26 para 24, 22 e 20\%. Oliveira (2001) encontrou maior peso vivo aos 49 dias de idade para as fêmeas em todos os tratamentos. Os pesos vivos de machos e de fêmeas observados no presente trabalho são maiores que aqueles citados para as codornas da linhagem italiana por Garcia (2002) de 177 e 209 g, respectivamente, para machos e fêmeas aos 49 dias de idade. Estes resultados diferem daqueles mencionados por Taboada et al. (1998), citados por Garcia (2002), que comparando o rendimento percentual de carne, músculos, pernas, peito, pescoço, coração e moela de codornas japonesas machos e fêmeas, não encontraram efeito significativa do sexo para estas variáveis.

O peso da carcaça dos machos não foi afetado pelo PN ( $\mathrm{P}>0,05)$, mas o peso da carcaça das fêmeas foi pior nos planos de nutrição 3 e 5 (Tabela 3) em comparação com os resultados dos demais planos $\left(\mathrm{P}_{\leq} 0,05\right)$, enquanto que, o rendimento de carcaça dos machos foi superior $(\mathrm{P}>0,05)$ ao das fêmeas. O resultado de peso de carcaça neste experimento não corrobora aquele de Oliveira (2001) que não observou efeito dos níveis de PB de 20, 22, 24 e $26 \%$, mas concorda com o maior rendimento de carcaça para os machos em comparação com as fêmeas também foi observado neste trabalho. As fêmeas apresentaram maior peso de carcaça (P d" 0,05$)$ nos planos de nutrição 1 (28 e $24 \%$ PB) e 4 (25,2 e $21,6 \%$ PB+L) e, menor no plano de nutrição 5 (22,4 e 19,2\%PB + L). Oliveira (2001) também observou maior peso para a carcaça das fêmeas em relação aos machos, independente, da redução da PB da dieta de 26 para 24,22 e $20 \%$ de proteína de 1 a 49 dias de idade. Somente o peso do peito das codornas fêmeas foi influenciado $(\mathrm{P} \leq 0,05)$ pelo $\mathrm{PN}$, sendo os melhores resultados obtidos nos PN 1; 2; 4; 6; 7; 8 e 9 em relação ao resultado do $\mathrm{PN}_{5}$. Enquanto, o peso do peito das fêmeas foi superior $\left(\mathrm{P}_{\leq} 0,05\right)$ ao dos machos nos $\mathrm{PN} 1 ; 6 ; 8$ e 9, e menor no $\mathrm{PN}_{6}$, o rendimento de peito não foi afetado (P > 0,05), conforme dados na Tabela 4 .

$\mathrm{O}$ peso de pernas dos machos foi melhor no $\mathrm{PN}_{2} \mathrm{em}$ relação ao $\mathrm{PN}_{7}$, enquanto para as fêmeas o pior peso de pernas foi observado no $\mathrm{PN}_{5}$, comparado aos outros $\mathrm{PN}$ $(\mathrm{P} \leq 0,05)$. As fêmeas mostraram maior peso de pernas nos $\mathrm{PN}_{1}$ e $\mathrm{PN}_{7}$, e menor que os machos no $\mathrm{PN}_{5}$ (Tabela 4), enquanto os piores rendimentos de pernas foram observados no $\mathrm{PN}_{7}$ e $\mathrm{PN}_{9}$ em comparação com os $\mathrm{PN}_{2} \mathrm{e}$ $\mathrm{PN}_{5}(\mathrm{P} \leq 0,05)$.

Considerando os efeitos principais, observou-se que o plano de nutrição não alterou os rendimentos de carcaça e de peito (Tabelas 3 e 4), mas alterou o rendimento de pernas $(\mathrm{P} \leq 0,05)$, que foi melhor nos planos de nutrição contendo valor protéico intermediário $\mathrm{PN}_{2}, \mathrm{PN}_{3}, \mathrm{PN}_{4}$ e $\mathrm{PN}_{5}$. No $\mathrm{PN}_{5}$. Portanto, observa-se que os tratamentos que resultaram nos piores consumos de ração e de ganho de peso, apresentaram melhor rendimento de pernas, o que sugere que o desenvolvimento desta variável é menos afetado pela redução do nível de proteína e da qualidade da dieta (Tabela 4), provavelmente pela alta proporção de ossos em relação aos músculos nesta região do corpo da codorna em comparação com, por exemplo, frangos de corte.

Apesar do maior peso vivo das fêmeas, os machos apresentaram maior rendimento de carcaça $\left(\mathrm{P}_{\leq} 0,05\right)$ e, as retiradas de ovário e oviduto durante a evisceração da carcaça das fêmeas, que não estão presentes na carcaça dos machos, além da maior proporção de fígado e de lipídeos nas vísceras não comestíveis das fêmeas, é a principal explicação para este resultado. Dados semelhantes foram descritos por Oliveira (2001), trabalhando com níveis crescentes de proteína de 20 até $26 \%$, em que o peso vivo das fêmeas foi superior ao dos machos, (225,3a vs 178,8b), mas o rendimento de carcaça foi inferior $(65,7 \mathrm{~b}$ vs 73,8a). Este resultado também apóia aquele de Fuentes et al. (2003) que fizeram semelhante observação ao estudarem níveis crescentes de proteína 
para codornas. Embora não-significativo, o maior peso e menor rendimento de carcaça para as fêmeas da linhagem italiana em relação aos machos também foi reportado por Garcia (2002). O maior rendimento de fígado nas fêmeas (2,41 vs. $1,90 \%$ ), reflete o possível aumento da síntese hepática de gordura em resposta à aproximação do ciclo de postura (Tabela 5). Estes lipídeos seriam exportados para o ovário para garantir o crescimento das múltiplas gemas em formação.

Taboada et al. (1998), citados por Garcia (2002), também constataram maior porcentagem de fígado nas fêmeas em comparação com os machos. No entanto, Oliveira (2001) não verificou efeito significativo da proteína da ração $(26,24,22$ e 20\%) sobre o rendimento de vísceras comestíveis (coração, fígado e moela) em codornas aos 49 dias de idade.

Os resultados deste trabalho indicaram que até 42 dias de idade, a deficiência protéica da ração deve afetar mais os pesos vivo, de carcaça e de peito de codornas fêmeas. A possível menor tolerância dessas aves às reduções no conteúdo e qualidade da proteína da ração, sugere a realização de pesquisas para estudar o manejo alimentar de codornas por separação de sexo, em que os machos poderiam receber rações, possivelmente, menos protéicas.

TABELA 4 - Pesos e porcentagens de peito e de pernas em função do plano de nutrição (PN) e do sexo de codornas criadas para a produção de carne.

\begin{tabular}{|c|c|c|c|c|c|c|}
\hline \multirow[b]{2}{*}{ PN } & \multicolumn{2}{|c|}{ Peito (g) } & \multirow{2}{*}{$\frac{\text { Peito (\%) }}{\text { Média }}$} & \multicolumn{2}{|c|}{ Pernas (g) } & \multirow{2}{*}{$\frac{\text { Pernas }(\%)}{\text { Média }}$} \\
\hline & Machos & Fêmeas & & Machos & Fêmeas & \\
\hline $\mathrm{PN}_{1}$ & $68,06^{\mathrm{B}}$ & $74,89^{\mathrm{aA}}$ & 40,20 & $43,43^{\mathrm{abB}}$ & $48,49^{\mathrm{aA}}$ & $25,86^{\text {bcd }}$ \\
\hline $\mathrm{PN}_{2}$ & 66,82 & $64,62^{\mathrm{ab}}$ & 39,13 & $47,64^{\mathrm{a}}$ & $45,41^{\mathrm{a}}$ & $27,72^{\mathrm{a}}$ \\
\hline $\mathrm{PN}_{3}$ & 63,42 & $61,28^{b c}$ & 39,27 & $43,87^{\mathrm{ab}}$ & $42,53^{\mathrm{a}}$ & $27,78^{\mathrm{ab}}$ \\
\hline $\mathrm{PN}_{4}$ & 61,18 & $67,90^{\mathrm{ab}}$ & 38,35 & $44,78^{\mathrm{ab}}$ & $47,10^{\mathrm{a}}$ & $27,30^{\mathrm{ab}}$ \\
\hline $\mathrm{PN}_{5}$ & $63,74^{\mathrm{A}}$ & $54,85^{\mathrm{cB}}$ & 40,00 & $44,87^{\mathrm{abA}}$ & $37,80^{\mathrm{bB}}$ & $27,94^{\mathrm{a}}$ \\
\hline $\mathrm{PN}_{6}$ & $61,48^{\mathrm{B}}$ & $68,38^{\mathrm{abA}}$ & 37,38 & $44,46^{\mathrm{ab}}$ & $46,27^{\mathrm{a}}$ & $26,10^{\text {bcd }}$ \\
\hline $\mathrm{PN}_{7}$ & 60,63 & $64,83^{\mathrm{ab}}$ & 37,48 & $39,70^{\mathrm{bB}}$ & $45,52^{\mathrm{aA}}$ & $25,46^{\mathrm{d}}$ \\
\hline $\mathrm{PN}_{8}$ & $60,16^{\mathrm{B}}$ & $69,56^{\mathrm{abA}}$ & 38,97 & $41,86^{\mathrm{ab}}$ & $44,44^{\mathrm{a}}$ & $25,98^{\text {bcd }}$ \\
\hline $\mathrm{PN}_{9}$ & $63,41^{\mathrm{B}}$ & $70,77^{\mathrm{abA}}$ & 38,83 & $42,11^{\mathrm{ab}}$ & $43,03^{\mathrm{a}}$ & $24,66^{\mathrm{d}}$ \\
\hline Machos & \multicolumn{2}{|c|}{63,21} & 38,44 & \multicolumn{2}{|c|}{43,64} & 26,54 \\
\hline Fêmeas & \multicolumn{2}{|c|}{66,34} & 39,25 & \multicolumn{2}{|c|}{44,51} & 26,41 \\
\hline \multicolumn{7}{|l|}{ Anova } \\
\hline PN & \multicolumn{2}{|c|}{$* *$} & ns & \multicolumn{2}{|c|}{$* *$} & $* *$ \\
\hline $\operatorname{Sexo}(\mathrm{S})$ & \multicolumn{2}{|c|}{$* *$} & $\mathrm{~ns}$ & \multicolumn{2}{|c|}{ ns } & ns \\
\hline$P N * S$ & \multicolumn{2}{|c|}{$* *$} & ns & \multicolumn{2}{|c|}{$* *$} & ns \\
\hline $\mathrm{CV}(\%)$ & \multicolumn{2}{|c|}{5,322} & 3,550 & \multicolumn{2}{|c|}{4,400} & 3,050 \\
\hline
\end{tabular}

${ }^{1} \mathrm{PN}=$ Plano de nutrição $1=28 \% \mathrm{~PB}, 1,5 \%$ lisina $(\mathrm{L})$ e $1,1 \%$ metionina+cistina $(\mathrm{MC})$ de 1 a 21 dias e $24 \% \mathrm{~PB}, 1,3 \% \mathrm{~L}$ e $0,9 \% \mathrm{MC}$ de 22 a 42 dias; $\mathrm{PN}_{2}=25,2 \% \mathrm{~PB}, 1,23 \% \mathrm{~L}$ e $0,83 \% \mathrm{MC}$ de 1 a 21 dias e de $21,6 \% \mathrm{~PB}, 1,0 \%$ L e $0,74 \% \mathrm{MC}$ de 22 a 42 dias; $\mathrm{PN}_{3}=22,4 \% \mathrm{~PB}, 0,71 \% \mathrm{MC}$ de 1 a 21 dias e 1,2\% L e 19,2\% PB, 0,64\% MC e 0,95\% L de 22 a 42 dias; $\mathrm{PN}_{4}=$ $\mathrm{PN}_{2}+\mathrm{L} ; \mathrm{PN}_{5}=\mathrm{PN}_{3}+\mathrm{L} ; \mathrm{PN}_{6}=\mathrm{PN}_{2}+\mathrm{MC} ; \mathrm{PN}_{7}=\mathrm{PN}_{3}+\mathrm{MC} ; \mathrm{PN}_{8}=\mathrm{PN}_{2}+\mathrm{L}+\mathrm{MC} ;$ e $\mathrm{PN}_{9}=\mathrm{PN}_{3}+\mathrm{L}+\mathrm{MC}$

${ }^{a b c}$ Médias nas colunas seguidas de letras minúsculas distintas dentro dos efeitos principais são diferentes pelo teste SNK $\left(\mathrm{P}_{\leq} 0,05\right)$.

${ }^{\mathrm{AB}}$ Médias nas linhas seguidas de letras maiúsculas distintas são diferentes pelo teste $\mathrm{F}(\mathrm{P} \leq 0,05)$.

$*\left(\mathrm{P}_{\leq} 0,01\right)$.

Ciênc. agrotec., Lavras, v. 31, n. 2, p. 514-522, mar./abr., 2007 
TABELA 5 - Peso e porcentagem de coração e de fígado em função do plano de nutrição (PN) e do sexo de codornas criadas para a produção de carne.

\begin{tabular}{|c|c|c|c|c|}
\hline PN & Coração (g) & Coração (\%) & Fígado (g) & Fígado (\%) \\
\hline $\mathrm{PN}_{1}$ & $2,02^{\mathrm{ab}}$ & $0,80^{\mathrm{bc}}$ & $5,25^{\mathrm{ab}}$ & 2,04 \\
\hline $\mathrm{PN}_{2}$ & $2,17^{\mathrm{ab}}$ & $0,90^{\mathrm{a}}$ & $5,87^{\mathrm{a}}$ & 2,40 \\
\hline $\mathrm{PN}_{3}$ & $2,05^{\mathrm{ab}}$ & $0,92^{\mathrm{a}}$ & $4,84^{\mathrm{ab}}$ & 2,17 \\
\hline $\mathrm{PN}_{4}$ & $2,08^{\mathrm{ab}}$ & $0,88^{\mathrm{ab}}$ & $4,61^{\mathrm{ab}}$ & 1,92 \\
\hline $\mathrm{PN}_{5}$ & $1,88^{b}$ & $0,91^{\mathrm{a}}$ & $4,30^{\mathrm{b}}$ & 2,10 \\
\hline $\mathrm{PN}_{6}$ & $1,92^{\mathrm{ab}}$ & $0,77^{\mathrm{c}}$ & $5,33^{\mathrm{ab}}$ & 2,13 \\
\hline $\mathrm{PN}_{7}$ & $2,24^{\mathrm{a}}$ & $0,93^{\mathrm{a}}$ & $5,42^{\mathrm{ab}}$ & 2,22 \\
\hline $\mathrm{PN}_{8}$ & $2,08^{\mathrm{ab}}$ & $0,86^{\mathrm{abc}}$ & $5,19^{\mathrm{ab}}$ & 2,12 \\
\hline $\mathrm{PN}_{9}$ & $2,25^{\mathrm{a}}$ & $0,93^{\mathrm{a}}$ & $5,53^{\mathrm{ab}}$ & 2,26 \\
\hline Machos & 2,10 & $0,92^{\mathrm{a}}$ & $4,33^{b}$ & $1,90^{\mathrm{b}}$ \\
\hline Fêmeas & 2,05 & $0,83^{b}$ & $5,97^{\mathrm{a}}$ & $2,41^{\mathrm{a}}$ \\
\hline \multicolumn{5}{|l|}{ Anova } \\
\hline $\mathrm{PN}$ & $* *$ & $* *$ & $* *$ & ns \\
\hline $\operatorname{Sexo}(\mathrm{S})$ & ns & $* *$ & $* *$ & $* *$ \\
\hline $\mathrm{PN}^{*} \mathrm{~S}$ & ns & ns & ns & ns \\
\hline $\mathrm{CV}(\%)$ & 6,072 & 6,752 & 11,290 & 10,180 \\
\hline
\end{tabular}

${ }^{1} \mathrm{PN}=$ Plano de nutrição $1=28 \% \mathrm{~PB}, 1,5 \%$ lisina $(\mathrm{L})$ e $1,1 \%$ metionina+cistina (MC) de 1 a 21 dias e $24 \% \mathrm{~PB}, 1,3 \% \mathrm{~L}$ e $0,9 \% \mathrm{MC}$ de 22 a 42 dias; $\mathrm{PN}_{2}=25,2 \% \mathrm{~PB}, 1,23 \% \mathrm{~L}$ e $0,83 \% \mathrm{MC}$ de 1 a 21 dias e de $21,6 \% \mathrm{~PB}, 1,0 \% \mathrm{~L}$ e $0,74 \% \mathrm{MC}$ de 22 a 42 dias; $\mathrm{PN}_{3}=22,4 \% \mathrm{~PB}, 0,71 \% \mathrm{MC}$ de 1 a 21 dias e $1,2 \% \mathrm{~L}$ e 19,2\% PB, 0,64\% MC e 0,95\% L de 22 a 42 dias; $\mathrm{PN}_{4}=$ $\mathrm{PN}_{2}+\mathrm{L} ; \mathrm{PN}_{5}=\mathrm{PN}_{3}+\mathrm{L} ; \mathrm{PN}_{6}=\mathrm{PN}_{2}+\mathrm{MC} ; \mathrm{PN}_{7}=\mathrm{PN}_{3}+\mathrm{MC} ; \mathrm{PN}_{8}=\mathrm{PN}_{2}+\mathrm{L}+\mathrm{MC} ; \mathrm{e} \mathrm{PN}_{9}=\mathrm{PN}_{3}+\mathrm{L}+\mathrm{MC}$

${ }^{a b c}$ Médias nas colunas seguidas de letras minúsculas distintas dentro dos efeitos principais são diferentes pelo teste SNK $\left(\mathrm{P}_{\leq} 0,05\right)$.

\section{CONCLUSÕES}

Para a fase de 1 a 21 recomendam-se $22,4 \%$ de $\mathrm{PB}$, $1,2 \%$ de lisina e $1,1 \%$ de aminoácidos sulfurosos (AAS), e de 22 a 42 sugerem-se 19,2\% de PB, 0,95\% de lisina e 0,9\% de AAS, sem deprimir os pesos da carcaça e de peito de machos e fêmeas de codornas à idade de abate.

Os machos apresentam maior rendimento de carcaça em virtude das fêmeas apresentarem maior perda de vísceras não comestíveis, portanto, recomenda-se o abate das mesmas em idade mais jovem (28 dias) que os machos (42 dias).

\section{REFERÊNCIAS BIBLIOGRÁFICAS}

DALMAU, A. B. Sistemas produtivos de codornices España. In: SIMPÓSIO INTERNACIONAL DE COTURNICULTURA, 1., 2002, Lavras. Anais... Lavras: NECTA/DZO/UFLA, 2002. p. 49-65.
FUENTES, M.; ZAPATA, J.; FREITAS, A. C. The effect of sex dietary protein and energy levels on quail carcass composition. In: INTERNATIONAL CONGRESS OF MEAT SCIENCE AND TECHNOLOGY, 49., 2003, Campinas. Proceedings... Campinas: ITAL, 2003. p. 515516.

GARCIA, E. A. Codornas para produção de carnes. In: SIMPÓSIO INTERNACIONAL DE COTURNICULTURA, 1., 2002, Lavras. Anais... Lavras: NECTA/DZO/UFLA, 2002. p. 97-108.

LEESON, S. et al. Commercial poultry nutrition. 2. ed. Guelph: Guelph University, 1997.

MURAKAMI, A. E. et al. Produção de codornas japonesas. Jaboticabal: Funep, 1998. 
OLIVEIRA, E. G. Pontos críticos no manejo e nutrição de codornas. In: SIMPÓSIO SOBRE MANEJO E NUTRIÇÃO DE AVES E SUÍNOS E TECNOLOGIA DE PRODUÇÃO DE RAÇÕES, 1., 2001, Campinas. Anais... Campinas: CBNA, 2001. p. 71-96.

OLIVEIRA, N. T. E. et al. Exigências de energia e proteína para codornas japonesas machos criadas para produção de carne. In: REUNIÃO ANUAL DA SOCIEDADE BRASILEIRA DE ZOOTECNIA, 37., 2000, Viçosa. Anais... Viçosa: UFV, 2000. p. 37.
PANDA, B.; SINGH, R. P. Developments in processing quail meat an eggs. World's Poultry Science Journal, Ithaca, v. 46, n. 11, p. 219-234, 1990.

SHRISVASTAV, A. K.; PANDA, B. A. A review of quail nutrition research in India. World's Poultry Science Journal, Ithaca, v. 55, n. 3, p. 73-81, 1999.

UNIVERSIDADE FEDERAL DE VIÇOSA. Sistema de análises estatísticas e genéticas (SAEG). Viçosa, 1983. 